\title{
I Social Media vanno all'università? Un'indagine sulle pratiche didattiche degli accademici italiani
}

\section{Stefania Manca ${ }^{1}$ - Maria Ranieri ${ }^{2}$}

${ }^{1}$ Consiglio Nazionale delle Ricerche (CNR), Istituto per le Tecnologie Didattiche

${ }^{2}$ Università degli Studi di Firenze, Dipartimento di Scienze della Formazione e Psicologia doi: 10.7358/ecps-2014-010-manc stefania.manca@itd.cnr.it maria.ranieri@unifi.it

\section{DO SOCIAL MEDIA GO TO UNIVERSITY? \\ A SURVEY OF ITALIAN SCHOLARS' TEACHING PRACTICES}

\section{Abstract}

This paper reports the results of a survey addressed to Italian academic scholars with the aim of identifying the teaching uses of Social Media in the field of university teaching practices. To this end, a survey instrument, already administered in the USA, was translated and adapted, and then sent to the entire Italian university scholar population. A total of 6.139 university researchers/teachers answered the survey, corresponding to 10,5\% of the population. The results show that Social Media use is still rather limited, for various reasons, and highlight the prevailing forms of teaching, mainly face-to-face based, over social and collaborative practices. Moreover, they also reveal factors of cultural resistance or of perception of uselessness of these tools. The latter may contribute to cause distress of the traditional models of the pedagogical relationship. A combination of factors of a pedagogical, pragmatic and value nature seems to lead teachers to prefer face-to-face teaching or, at most, traditional e-learning platforms. However, the overall results emphasize ambivalent attitudes towards the benefits and challenges of these tools in the context of higher education, as already pointed out by the literature.

Keywords: Higher education, Italian university, Pedagogical relationship, Social Media, Teaching practice. 


\section{INTRODUZIONE}

Se fino alla metà degli anni 2000 l'espressione e-learning ha rappresentato un termine chiave nella letteratura sulla formazione a distanza, a partire dagli anni successivi nuove espressioni si sono imposte nel lessico relativo agli studi sull'apprendimento basato sul web (web-based learning). Si comincia a parlare di Personal Learning Environment (Anderson, 2006; Fini \& Cigognini, 2009), di e-learning 2.0 (Downes, 2005; Bonaiuti, 2006) e, più recentemente, di Massive Open Online Courses o MOOCs (de Waard et al., 2011) per segnalare il passaggio dall'e-learning di prima generazione, basato sull'uso di piattaforme chiuse (i cosiddetti Learning Management System), all'e-learning di seconda generazione, caratterizzato dall'impiego di dispositivi del web 2.0. Questi ultimi vengono descritti di solito come «dinamici», «interattivi», «democratici», «sociali», "centrati sull'utente»: se la prima generazione del web si distingueva, infatti, per l'accesso e la fruizione di risorse, la seconda si caratterizza per la produzione e socializzazione immediata dei contenuti secondo l'ormai nota formula del user-generated content (O'Reilly, 2005).

I Social Media, che costituiscono l'oggetto specifico di questo contributo, appartengono alla seconda generazione del web e, negli ultimi anni, sono stati visti da più parti come un potente volano per la trasformazione delle pratiche di insegnamento e apprendimento in senso aperto, interattivo e sociale (Solomon \& Schrum, 2010; Siemens \& Weller, 2011; Fedeli, 2012). In particolare, considerando il contesto universitario, diversi autori (cfr. Brown \& Adler, 2008) hanno evidenziato come l'adozione di questi dispositivi produca o richieda un radicale cambio di paradigma pedagogico con conseguenze "rivoluzionare» per le istituzioni accademiche. Bisogna, tuttavia, osservare che, mentre abbondano lavori di riflessione sulle potenzialità dei Social Media per l'apprendimento (Anderson, 2009; Siemens \& Weller, 2011) o ricerche a carattere empirico sugli usi che le nuove generazioni ne fanno in ambito accademico (cfr. Davis III, Deil-Amen, Rios-Aguila, \& Sacramento Gonzales Canche, 2012), molto meno comuni sono le riflessioni o le indagini sulle pratiche digitali degli accademici (cfr. Jaschik \& Lederman, 2013; Allen \& Seaman, 2014). Come osserva Brown (2012, p. 50), «Sono rare le discussioni situate nel contesto sul potenziale del Web 2.0 per la didattica. Più comuni sono le analisi generalizzate dei benefici del Web 2.0 nel settore universitario".

Eppure un'attenzione specifica agli usi effettivi che gli accademici fanno dei Social Media nella loro attività didattica e una maggiore comprensione delle percezioni che hanno nei riguardi di questi dispositivi consentirebbero di superare il "genericismo" che spesso caratterizza le riflessioni intorno al ruolo delle ICT nella didattica universitaria. 
Il presente lavoro intende offrire un contribuito di carattere empirico alla ricerca sulle pratiche digitali degli universitari italiani, focalizzandosi in particolare sui Social Media e prestando attenzione agli usi e alle percezioni che gli accademici hanno circa il potenziale di questi strumenti per la didattica. Nella prima parte del contributo viene presentato il quadro teorico e metodologico che ha guidato l'indagine, mentre nella seconda parte vengono illustrati e discussi i risultati. L'articolo si chiude con alcune considerazioni sulle future aree di ricerca nel settore.

\section{QUADRO TEORICO DI RIFERIMENTO}

Nel dibattito attuale sul ruolo dei Social Media per l'apprendimento sono soprattutto gli esponenti del connettivismo (Siemens, 2005) e dell'approccio del social learning (Anderson, 2009) ad averne evidenziato le potenzialità pedagogiche. Secondo il connettivismo, la conoscenza e l'apprendimento sono distribuiti nelle reti interpersonali e l'apprendimento consiste nella capacità di attraversare tali reti, di connettersi ad esse e promuoverle. In questa prospettiva, il «sapere dove» trovare le informazioni è più importante del «sapere cosa» cercare. Si tratta, pertanto, di imparare a muoversi tra una varietà di ambiti e di interagire con una vasta rete di relazioni e risorse, riuscendo a costruire e gestire la conoscenza, collegandola ai concetti esistenti e applicandola ai diversi contesti. Tali capacità sono meglio sviluppate attraverso forme di apprendimento partecipativo, che comportano la costruzione di comunità di conoscenza all'interno delle quali gli studenti stessi individuano gli obiettivi di apprendimento condivisi e decidono quali sono gli strumenti concettuali funzionali allo sviluppo dell'attività.

I Social Media, e più in generale gli applicativi del web 2.0, vengono visti come dispositivi in grado di facilitare l'apprendimento partecipativo e la costruzione di conoscenza (Collis \& Moonen, 2008). Essendo interattivi, sociali e flessibili, possono fornire agli studenti uno spazio in cui collaborare per generare attivamente conoscenza, piuttosto che limitarsi a ricevere passivamente informazioni (Brown \& Adler, 2008). Queste forme di collaborazione non si limitano al lavoro in piccoli gruppi, ma possono riguardare interi network costituiti da una pluralità di individui uniti da un interesse comune, i cosiddetti "collettivi» (Dron \& Anderson, 2009). Parallelamente, le nuove generazioni di studenti vengono rappresentate come sempre meno soddisfatte di limitarsi alla fruizione passiva dei contenuti e sempre più propense alla creazione e condivisione di risorse digitali (Tapscott \& Williams, 2007). In questo contesto, alcuni autori (Yu, Tian, Vogel, \& Kwok, 2010) 
sostengono che un maggiore utilizzo dei Social Media in ambito accademico possa contribuire a riconnettere istituzioni universitarie e nuove generazioni; altri (Downes, 2010) ritengono, invece, che le istituzioni accademiche siano destinate ad un drastico crollo di fiducia da parte dei propri utenti.

Rispetto a queste visioni che enfatizzano il potenziale dei Social Media e che alcuni autori qualificano come essenzialistiche (Brown, 2012), non mancano posizioni più caute, se non addirittura critiche, che richiamano l'importanza di considerare le esigenze e i vincoli tipici dei contesti formativi. Selwyn (2011), ad esempio, sottolinea che non si può dare per scontato che da pratiche non educative possano derivare automaticamente benefici di carattere educativo, come se tutto fosse educazione; né si può assumere che gli studenti utilizzino o intendano utilizzare i Social Media per attività di studio. Oltretutto, l'idea stessa dei Social Media come strumenti partecipativi e democratici non trova adeguati riscontri nella realtà osservata, nella quale in pochi sono effettivamente partecipanti attivi e/o produttori di contenuti (Hargittai \& Walejko, 2008). I più critici si spingono ad evidenziare come un uso intensivo dei Social Media possa addirittura condurre ad un impoverimento culturale e cognitivo (Carr, 2010).

Infine, altri autori mettono, invece, in guardia rispetto a possibili tensioni. Conole (2013) fa luce sui rischi derivanti dalla moltiplicazione e frammentazione dei canali informativi che possono generare disorientamento e richiedono, pertanto, lo sviluppo di adeguate strategie di selezione e validazione di risorse. Crook (2008) indica ulteriori motivi di tensione, tra i quali la sfida della ridefinizione dei ruoli di docente e studente, la contrapposizione tra gruppo di lavoro chiuso che opera in una tradizionale piattaforma e-learning e unarena di lavoro aperta ai contribuiti e ai commenti di tutti, il rapporto tra apprendimento individuale e apprendimento collaborativo, laddove quest'ultimo solleva problemi non solo ai fini della valutazione ma anche in considerazione dei particolari stili di apprendimento degli studenti. A queste criticità se ne aggiungono altre che riguardano specificamente il contesto universitario. In generale, si può evidenziare una profonda diversità di valori e visioni tra mondo accademico e mondo social: il primo si caratterizza per un'organizzazione di tipo verticale e gerarchico, mentre il secondo per approcci orizzontali e partecipativi; il processo formale di revisione e valutazione scientifica tipico del primo viene messo in discussione dalla produzione di contenuti "dal basso" tipico del secondo; e ancora il concetto di elaborati sempre in progress soggetti a revisioni e aggiornamenti continui contrasta con l'esigenza delle istituzioni accademiche di individuare e presentare agli studenti un corpus consolidato di materiali e argomenti (Esposito, 2011).

Passando dalle visioni generali all'analisi delle pratiche specifiche e delle rappresentazioni che gli accademici hanno dei Social Media, emergono 
ulteriori elementi di interesse. Da una ricerca di Ajjan e Hartshorne (2008) risulta che la maggior parte dei docenti coinvolti nello studio ha una opinione favorevole verso l'integrazione dei Social Media nella didattica. Ciononostante, quasi nessuno ne fa uso o ha intenzione di usarli. Tra i motivi indagati all'origine di questa scarsa propensione ad avvalersene due ragioni emergono come particolarmente significative. Da un lato, limitata è la percezione di utilità e compatibilità di questi strumenti con le pratiche correnti e i valori ad esse sottesi; dall'altro, il senso di inadeguatezza nell'uso di questi dispositivi (scarso senso di autoefficacia) e la mancanza di buone pratiche sembrano influire pesantemente sulla decisione di non avvalersene.

Altri autori riconducono la limitata propensione all'adozione effettiva ad una più generale resistenza al cambiamento pedagogico richiesto dall'uso delle nuove tecnologie, alla mancanza di fiducia in se stessi e ad una incertezza legata al cambiamento in generale (Collis \& Moonen, 2008). Così, ad esempio, Ravenscroft (2009) argomenta che gli accademici tendono a sposare pedagogie che vincolano e contengono il potenziale in qualche modo anarchico dei Social Media optando per piattaforme chiuse di prima generazione che siano espressione di approcci pedagogici centrati sul docente, piuttosto che sul contributo dello studente e sul ruolo del networking.

All'opposto, da altri studi emerge che non c'è correlazione tra la disponibilità ad adottare pedagogie centrate sullo studente e l'autoapprendimento e l'adozione di tecnologie nella didattica (cfr. Ulrich \& Karvonen, 2011). Altri fattori sembrano più decisivi: in particolare, Veletsianos e Kimmons (2013) sottolineano come l'erosione dei confini tra identità personale e identità professionale, i bassi livelli di controllo consentiti, in particolare, dai social network nella gestione delle relazioni e la mancanza di competenze digitali e formazione ostacolino pesantemente l'utilizzo di tali tecnologiche in ambito accademico. Più in generale, Brown (2012) sostiene che le interpretazioni centrate sulla resistenza al cambiamento non tengono conto dei concreti contesti d'uso e delle pratiche effettive sulle quali influiscono le credenze degli accademici circa la loro specifica attività di insegnamento, i loro obiettivi in questi contesti, la loro interpretazione dei bisogni degli studenti, la loro identità in quanto docenti e le opinioni dei colleghi. Poiché gli accademici svolgono una funzione cruciale di mediazione, le loro percezioni sul potenziale dei Social Media dovrebbero essere viste come una potente risorsa per meglio comprendere come possano essere usati in modo diversificato rispetto ai differenti contesti o aspetti di quel contesto. 


\section{I SOCIAL MEDIA OGGETTO DELL'INDAGINE}

Benché non esistano modalità univoche di classificazione, i Social Media sono applicazioni del web 2.0 che includono dispositivi quali i blog, i wiki, i siti di social networking (SNS), i servizi di condivisione di immagini o video, e qualunque medium il cui funzionamento si basi sulla partecipazione attiva degli utenti. Questi media, infatti, abilitano e al tempo stesso si nutrono della partecipazione delle persone che, attraverso le loro azioni di produzione, condivisione, scambio e interazione, popolano questi ambienti virtuali di contenuti e relazioni sociali (cfr. Mason \& Rennie, 2008). Di seguito vengono brevemente presentati quegli strumenti che sono stati specifico oggetto di indagine nel presente studio.

\subsection{Twitter}

Lanciato nel 2006, Twitter è un sito di microblogging che consente la pubblicazione di piccoli contenuti sotto forma di messaggi di testo (fino a $140 \mathrm{ca}-$ ratteri), immagini, video, audio musicali, segnalazione di link, ecc., che vengono pubblicati in un servizio di social network visibili a tutti o soltanto ai membri della propria rete personale. Twitter, che conta oggi più di $600 \mathrm{mi}-$ lioni di utenti attivi, ha attratto l'attenzione degli studiosi di comunicazione mediale, di psicologia dei comportamenti sociali, degli esperti di fenomeni politici, ma anche di educatori e professionisti della formazione. Numerose sono, infatti, anche le esperienze di apprendimento formale e informale che si sono avvalse dell'uso di Twitter (cfr. Gao, Luo, \& Zhang, 2012).

\subsection{Facebook}

Con il più alto numero di iscritti, oltre un miliardo e 300 milioni (Facebook, 2014), Facebook è considerato il social network per antonomasia. Creato nel 2004, disponibile in più di 70 lingue, Facebook più che un sito di social network è in realtà una piattaforma sociale che contiene al proprio interno una miriade di funzionalità e di applicazioni sia native che sviluppate da terze parti (gruppi, pagine, messaggistica privata, album di foto e video, note, eventi, ma anche giochi, applicazioni professionali). La crescente diffusione di Facebook anche come strumento e ambiente di apprendimento è testimoniata dal numero crescente di esperienze didattiche e di ricerca: accanto a un utilizzo di supporto a pratiche accademiche istituzionali o come ambiente di sostegno per lo sviluppo professionale (Ranieri \& Manca, 2013b), si sta in- 
fatti diffondendo un uso più didattico ed educativo di Facebook nei contesti di apprendimento formale e informale (Manca \& Ranieri, 2013; Ranieri \& Manca, 2013a; Greenhow, Gleason, \& Li, 2014).

\subsection{LinkedIn}

Forse il più noto tra i social network professionali, con quasi 300 milioni di utenti LinkedIn è diventato uno strumento ampiamente usato per favorire lo scambio di chi cerca e offre lavoro in molte realtà professionali. L'importanza di LinkedIn nelle procedure di selezione e di assunzione del personale nelle organizzazioni (Caers \& Castelyns, 2011) è stata ampiamente riconosciuta, fino a diventare anche uno spazio di sperimentazione per studenti impegnati a progettare il loro futuro lavorativo e professionale (Gerard, 2012) o nei percorsi di orientamento in combinazione con altri social network (Sansone, Cucchiara, \& Ligorio, 2013).

\subsection{Google+}

Google+ o Google Plus è l'ultimo sito di social network in ordine di tempo (2011), lanciato da Google. Il sito contiene importanti funzionalità di comunicazione multimediale quali le sessioni di comunicazione audio-video (gli hangouts) e altri servizi di condivisione. Il sistema dei contatti è organizzato e suddiviso in cerchie creabili e modificabili dall'utente, oltre a quelle già predefinite dal sistema. Per potersi registrare su Google+ occorre disporre di un account Google, il quale fornisce maggiori garanzie di altri servizi sull'identità dei profili. Negli ultimi tempi il numero di utenti è diventato quasi equivalente a quello di Facebook, anche se il numero di quelli realmente attivi è di molto inferiore.

\subsection{Podcast}

I podcast sono documenti in formato audio o video che possono essere fruiti online, attraverso delle applicazioni apposite, oppure scaricati e salvati nella memoria di un dispositivo per la successiva riproduzione. Una modalità di utilizzo frequente in ambito scolastico e universitario è quella di produrre i podcast delle lezioni che gli studenti potranno riascoltare in momenti successivi. Alcune università italiane mettono in linea interi archivi di lezioni registrate in forma di podcast, che possono essere scaricate e fruite anche da non 
studenti. Il servizio più noto è quello Apple di iTunes $U$, che grazie ad un'app apposita per iPad, iPhone e iPod touch mette a disposizione più di 500.000 lezioni, video, libri e altre risorse gratuite su migliaia di argomenti. Per l'uso nel contesto universitario, si rimanda a: O’Bannon, Lubke, Beard, \& Britt, 2011; Ng'ambi \& Lombe, 2012; Van Zanten, Somogyi, \& Curro, 2012.

\section{6. $B \log$}

I blog sono un particolare tipo di applicazioni Internet che consentono la pubblicazione di contenuti testuali o multimediali attraverso la logica dell'articolo o post, che vengono visualizzati secondo l'ordine cronologico in cui sono stati scritti. I blog sono stati il primo strumento di massa messo a disposizione dal web 2.0 che ha permesso, anche a chi avesse pochissime competenze tecniche, di pubblicare contenuti nuovi e originali sul web. In ambito educativo la loro efficacia è stata riconosciuta da molto tempo e la letteratura testimonia una loro diffusione in molteplici contesti didattici e di apprendimento (cfr. Churchill, 2009 e 2011; Paulus, Payne, \& Jahns, 2009). In ambito universitario il loro ruolo è stato indagato anche in connessione con l'uso di altri strumenti, quali i social network (Ivala \& Gachago, 2012) o i wiki (Avci \& Askar, 2012).

\subsection{Wiki}

I wiki sono ambienti web che consentono di comporre dei testi che possono essere integrati, modificati o cancellati attraverso un browser web, in genere utilizzando un linguaggio di markup semplificato o un editor di testo online. $\mathrm{Si}$ tratta in genere di documenti ipertestuali i cui contenuti vengono sviluppati in maniera collaborativa da tutti coloro che hanno accesso al database o repository su cui vengono immagazzinate le diverse versioni. L'importanza svolta dai wiki negli ambienti di apprendimento collaborativo è stata testimoniata da molteplici contributi, che ne hanno evidenziato il ruolo di amplificatori cognitivi della scrittura a più mani (Trentin, 2009; Woo, Chu, \& $\mathrm{Li}, 2013)$. Lo stesso esempio di wiki più noto, Wikipedia, che ha raggiunto nella sua versione italiana oltre un milione di voci, è stato oggetto di studio anche in relazione all'uso che ne viene fatto in ambito didattico e universitario (Cress, 2012; Knight \& Pryke, 2012). 


\subsection{YouTube e Vimeo}

YouTube è una piattaforma web che consente la condivisione e la visualizzazione di video attraverso un sistema di video-sharing. Una sezione consistente di video per usi didattici è costituita da YouTube EDU, che organizza i materiali per livello scolare, universitario o per il lifelong learning. Anche Vimeo mette a disposizione la possibilità di caricare produzioni multimediali originarie, purché i contenuti siano stati creati unicamente dall'utente. L'importanza che il ruolo fondamentale dell'uso dei video riveste nell'apprendimento è stata sottolineata da più parti, anche in riferimento a questi due ambienti e per la loro rilevanza rispetto a diversi ambiti disciplinari (Jaffar, 2012; Stohlmann, 2012).

\subsection{ResearchGate e Academia.edu}

ResearchGate e Academia.edu possono essere definiti come la versione di LinkedIn per gli scienziati, avendo infatti l'obiettivo di consentire ai ricercatori la condivisione delle loro pubblicazioni scientifiche e di seguire studiosi di tematiche specifiche. Entrambi partecipano al movimento dell'Open Science, che attraverso l'uso delle più recenti tecnologie digitali e delle reti sociali promuove un approccio aperto alla diffusione dei risultati della produzione scientifica (Peters, 2010). Globalmente permettono di gestire i documenti attraverso elenchi aggiornabili (in modo manuale o automatico) delle proprie pubblicazioni, oppure attraverso la ricerca su diversi database esterni e di aggiungere riferimenti bibliografici, o ancora attraverso la ricerca semantica di documenti basata sull'analisi del contenuto; inoltre, è possibile la creazione di gruppi accomunati da uno specifico interesse di ricerca (Codina, 2009).

\subsection{SlideShare}

SlideShare è un'applicazione che consente la condivisione di documenti testuali quali presentazioni Power Point, file PDF, o in altri formati open. Nato per condividere documenti tra membri della stessa organizzazione, il servizio si è ampliato fino a consentire l'accesso ed iscrizione a chiunque; inoltre, fornisce la possibilità di taggare i documenti attraverso apposite keyword, di valutare e commentare i contenuti inseriti da altri utenti e, analogamente al meccanismo della lista di amici/contatti dei social network, consente di seguire gli utenti ai cui contenuti si sia più interessati. 


\section{STRUMento E METOdologia Di SOMMINistrazione}

\subsection{Lo strumento}

Lo studio presenta i risultati di un'indagine effettuata attraverso la somministrazione di un questionario tradotto ed adattato a partire dal testo di una survey che la casa editrice internazionale Pearson, in collaborazione con il Babson Survey Research Group, somministra da alcuni anni ai docenti delle università degli Stati Uniti (Tinti-Kane, Seaman, \& Levy, 2010; Moran, Seaman, \& Tinti-Kane, 2011; Moran, Seaman, \& Tinti-Kane, 2012; Seaman \& Tinti-Kane, 2013). Lo scopo di queste indagini è quello di fare una ricognizione degli usi personali, didattici e professionali dei Social Media da parte del personale universitario con incarichi di insegnamento.

Per lo scopo dell'indagine che viene qui presentata si è scelto di tradurre e adattare per il contesto italiano il questionario che è stato somministrato nel 2012 (Moran et al., 2012). In particolare, è stato necessario modificare alcune domande preesistenti per colmare alcune differenze culturali e linguistiche tra il contesto statunitense e quello italiano (ad es., si è preferito rendere con "Piattaforma e-learning» la dicitura Learning Management System, che in Italia è nota quasi solo agli addetti ai lavori) o per aggiungere opzioni di risposta non contemplate nell'indagine originaria (ad es., dato che uno degli obiettivi era quello di investigare gli usi professionali dei Social Media, sono stati aggiunti social network accademici come ResearchGate e Academia.edu).

Inoltre, sono state introdotte nuove domande per dar conto delle specificità culturali-organizzative del contesto accademico italiano. Sono state, infine, aggiunte una serie di domande finalizzate a indagare in maniera più approfondita alcuni aspetti di utilizzo dei Social Media, relativamente ai tre ambiti di riferimento (personale, didattico e professionale). Nessuna delle domande presenti nella survey originaria (Moran et al., 2012) è stata eliminata, dal momento che uno degli obiettivi della ricerca era quello di operare il confronto dei risultati conseguiti nei due diversi contesti geografici.

Il questionario finale contiene alcune domande di carattere socio-anagrafico e professionale, una sezione di informazioni sull'ateneo in cui si insegna, una serie di sezioni dedicate a esplorare la frequenza, le motivazioni e le modalità d'uso di diversi strumenti per scopi personali, didattici e professionali. In particolare, si sono esplorate le ragioni che possono portare ad usare questi strumenti o, al contrario, ad inibirne l'uso attraverso una serie di fattori. L'articolazione interna completa del questionario può essere visualizzata nell'appendice del report dei risultati (Manca, 2014). 
Per poter operare il confronto tra le tre diverse tipologie d'uso, nella presentazione iniziale del questionario era precisato che questo si rivolgeva a coloro che, operando sia in università statali che non statali, ricoprissero anche incarichi di insegnamento. Nel presente studio vengono, tuttavia, presentati solo i risultati relativi alle domande sull'uso didattico dei Social Media (per una presentazione completa dei risultati, si rimanda a Manca, 2014).

Lo strumento, prima di essere somministrato, è stato testato da un piccolo gruppo di ricercatori afferenti a università diverse, con l'obiettivo di verificarne la comprensibilità e la completezza, oltre che per stimare i tempi di compilazione. Questi ultimi sono stati calcolati nella misura dei 20 minuti circa.

\subsection{Individuazione dei destinatari}

Per poter avere un quadro della situazione italiana il più ampio possibile si è deciso di raggiungere tutto il personale di ricerca di tutte le università, così come risultante in organico alla data di settembre 2013. I nominativi sono stati acquisiti attraverso le informazioni presenti sul portale del MIUR dedicato all'università (http://hubmiur.pubblica.istruzione.it/web/universita/ home). Da questi elenchi, scaricabili in formato Excel, che contengono informazioni relative alla fascia, la facoltà, il settore scientifico-disciplinare, le aree disciplinari e il dipartimento di afferenza, sono stati ricavati 57.590 nominativi.

Dal momento che nelle università telematiche il personale docente in organico è spesso sottodimensionato rispetto alle reali esigenze di copertura didattica, per cui ci si avvale frequentemente di professori a contratto, per non penalizzare la rappresentanza di questi atenei si è deciso di reperire i loro nominativi attraverso la consultazione dei siti web degli atenei, con la consapevolezza che fossero presenti eventuali lacune od omissioni. Sono stati, quindi, acquisiti ulteriori 585 nominativi che, sommati ai 57.590 già individuati, hanno prodotto un totale di 58.175 docenti/ricercatori.

Si è a questo punto proceduto al reperimento degli indirizzi di posta elettronica attraverso la consultazione dei siti web dei singoli atenei, che normalmente rendono disponibili pubblicamente gli indirizzi e-mail istituzionali del proprio personale anche attraverso apposite rubriche, al fine di poter inoltrare l'invito di partecipazione all'indagine. Qualora non direttamente rintracciabili nei siti web, gli indirizzi sono stati creati attraverso un algoritmo che poggiasse su quelli già noti (es.nome.cognome@dominio.it, n.cognome@dominio.it, ecc.). 


\subsection{Procedura di somministrazione e di raccolta dei dati}

Dopo essere stato testato, il questionario è stato messo online attraverso il software open source LimeSurvey (http://www.limesurvey.org/), che consente la creazione di identificativi personalizzati associati agli indirizzi e-mail. È stato, quindi, possibile controllare chi avesse accesso al questionario, escludendo la partecipazione di persone non incluse nel campione. Questa informazione è stata, però, tenuta scorporata dalle risposte, al fine di garantire l'anonimato dei compilatori. Chi era interessato a conoscere i risultati della ricerca e disponibile a essere contattato per ulteriori sviluppi poteva inserire il suo indirizzo e-mail nell'ultima pagina del questionario.

La compilazione è stata resa possibile dal 1 ottobre al 15 dicembre 2013. A metà di questo periodo di tempo è stato inviato un promemoria a quanti non avevano riempito il questionario fino a quel momento. Nella mail di invito che ciascuno riceveva era possibile decidere di non partecipare e di non essere ulteriormente contattati cliccando su un link apposito. Le rinunce di questo tipo sono state 959 .

\section{Risultati}

\subsection{Caratteristiche socio-demografiche e professionali}

Al termine del periodo di partecipazione all'indagine sono state raccolte 7.388 occorrenze di compilazione, di cui 5.632 complete e 1.756 incomplete. Di queste ultime sono state considerate solo quelle che avessero completato almeno la prima sezione di domande utili rispetto al tema dell'indagine, oltre a quelle a carattere socio-demografico e professionale, che sono risultate pari a 507. Il numero finale dei questionari ritenuti utili è stato di 6.139, con un tasso di risposta pari al 10,5\% degli inviti spediti.

Nei risultati che verranno presentati di seguito sono stati omessi i dati relativi a Google+ perché dalle risposte aperte e dai commenti si è evinto facilmente come molti dei rispondenti avessero frainteso il nome di questo social network con quello della famiglia dei prodotti Google.

Una prima analisi ha riguardato il confronto tra la distribuzione del campione e quella della popolazione; si è proceduto cioè a verificare che il campione presentasse caratteristiche sovrapponibili a quelle della popolazione rispetto ad alcune variabili socio-demografiche e professionali. Sono stati effettuati, quindi, dei test del Chi-quadrato per verificare che la stratificazione 
del campione riflettesse quella della popolazione rispetto alle variabili del genere (maschio, femmina), ruolo (ricercatore, professore associato, professore ordinario), area scientifico-disciplinare (Scienze matematiche e informatiche, Scienze naturali, Scienze applicate, Scienze umane, Arte e Spettacolo, Scienze sociali), tipologia di ateneo (statale, non statale; tradizionale, telematico), area geografica della sede universitaria (Nord, Centro, Sud, Isole). Dal momento che il test del Chi-quadrato ha come ipotesi nulla che la distribuzione osservata sia sovrapponibile con quella attesa, qualunque valore di significatività maggiore a 05 consente di supportare l'ipotesi nulla e quindi di concludere che il campione e la popolazione siano sovrapponibili rispetto a quella determinata variabile. Tuttavia, data l'elevata ampiezza campionaria, a fronte di un risultato statisticamente significativo $(p<, 05)$, il calcolo della dimensione dell'effetto, che fornisce una misura della sostanzialità dell'effetto, consente di valutare il reale scostamento tra le frequenze osservate e quelle attese (Chiorri, 2014). Questo significa che una dimensione dell'effetto trascurabile o molto bassa $(r<, 10-20)$ può indicare una sostanziale sovrapponibilità tra il campione e la popolazione anche in presenza di un valore di significatività maggiore a , 05 .

Tutte le analisi statistiche sono state effettuate con il software IBM SPSS 18.0. Come vedremo nel seguito, il test sulle variabili del genere, ruolo, settore disciplinare, tipologia di ateneo e area geografica ha consentito di supportare l'ipotesi della sovrapponibilità delle due distribuzioni. Solo il confronto tra atenei tradizionali e telematici ha evidenziato una sostanziale sovrarappresentazione dei docenti delle università telematiche.

Nella Tabella 1 sono riportati i dati relativi alla distribuzione del campione rispetto alle caratteristiche socio-demografiche del genere, l'età, gli anni di insegnamento, la tipologia di contratto, il tipo di contratto da dipendente e il ruolo. Quando disponibili, questi dati sono stati confrontati con quelli della popolazione. Il test del Chi-quadrato sulla variabile del genere ha evidenziato che le due distribuzioni presentano caratteristiche di stratificazione simili $\left(\mathrm{X}^{2}(1)=29,238, p<, 01, r=, 07\right)$, così come ad analoghi risultati ha condotto il test sulla variabile di ruolo $\left(\mathrm{X}^{2}(2)=103,921, p<, 01\right.$, $r=, 13)$.

Nella Tabella 2 sono presentati i dati relativi ai settori scientificodisciplinari, sia nel dettaglio che raggruppati per macro-aree. Anche in questo caso è stato possibile operare il confronto tra la distribuzione del campione e quella della popolazione. Il test del Chi-quadrato ha evidenziato che le due distribuzioni sono sovrapponili per quanto riguarda la variabile accorpata delle macro-aree scientifico-disciplinari $\left(\mathrm{X}^{2}(4)=134,370\right.$, $p<, 01, r=, 15)$. 
Tabella 1. - Caratteristiche socio-demografiche e professionali $(N=6.139)$.

\begin{tabular}{|c|c|c|c|}
\hline & & Campione & Popolazione $(\mathrm{N}=58.175)$ \\
\hline \multirow{2}{*}{ SESSO } & Maschi & $3.727(60,7 \%)$ & $37.245(64,0 \%)$ \\
\hline & Femmine & $2.412(39,3 \%)$ & $20.930(36,0 \%)$ \\
\hline \multirow{5}{*}{ ETÀ } & Meno di 25 & $0(0,0 \%)$ & - \\
\hline & $25-34$ & $253(4,1 \%)$ & - \\
\hline & $35-44$ & $1.939(31,6 \%)$ & - \\
\hline & $45-55$ & $2.185(35,6 \%)$ & - \\
\hline & $55+$ & $1.762(28,7 \%)$ & - \\
\hline \multirow{6}{*}{$\begin{array}{c}\text { ANNI } \\
\text { DI } \\
\text { INSEGNAMENTO }\end{array}$} & Meno di 1 & $52(0,8 \%)$ & - \\
\hline & Da 1 a 5 & $670(10,9 \%)$ & - \\
\hline & Da 6 a 9 & $1.047(17,1 \%)$ & - \\
\hline & Da 10 a 15 & $1.690(27,5 \%)$ & - \\
\hline & Da 16 a 20 & $822(13,4 \%)$ & - \\
\hline & $20+$ & $1.858(30,3 \%)$ & - \\
\hline \multirow{3}{*}{$\begin{array}{c}\text { Tipologia } \\
\text { DI } \\
\text { CONTRATTO }\end{array}$} & $\begin{array}{c}\text { Dip. universitario } \\
\text { a tempo indeterminato }\end{array}$ & $5.599(91,2 \%)$ & - \\
\hline & $\begin{array}{l}\text { Dip. universitario } \\
\text { a tempo determinato }\end{array}$ & $444(7,2 \%)$ & - \\
\hline & Professore a contratto & $96(1,6 \%)$ & - \\
\hline \multirow{2}{*}{$\begin{array}{c}\text { ContratTo } \\
\text { DA DIPENDENTE } \\
(\mathrm{N}=6043)\end{array}$} & Tempo parziale & $189(3,1 \%)$ & - \\
\hline & Tempo pieno & $5.854(96,9 \%)$ & - \\
\hline \multirow{3}{*}{$\begin{array}{c}\text { RuOLO } \\
(\mathrm{N}=6043)\end{array}$} & Ricercatore & $3.130(51,8 \%)$ & $26.824(46,6 \%)$ \\
\hline & Professore associato & $1.704(28,2 \%)$ & $16.086(28,0 \%)$ \\
\hline & Professore ordinario & $1.209(20,0 \%)$ & $14.612(25,4 \%)$ \\
\hline
\end{tabular}

Tabella 2. - Settori scientifico-disciplinari e macro-aree disciplinari $(N=6.139)$.

\begin{tabular}{lcc}
\hline & CAMPione & $\begin{array}{c}\text { Popolazione } \\
(\mathrm{N}=57.590)\end{array}$ \\
\hline 01. Scienze matematiche e informatiche & $435(7,1 \%)$ & $3.174(5,5 \%)$ \\
02. Scienze fisiche & $288(4,7 \%)$ & $2.257(3,9 \%)$ \\
03. Scienze chimiche & $307(5,0 \%)$ & $2.931(5,1 \%)$ \\
04. Scienze della Terra & $121(2,0 \%)$ & $1.056(1,8 \%)$ \\
05. Scienze biologiche & $500(8,1 \%)$ & $4.980(8,6 \%)$ \\
06. Scienze mediche & $653(10,6 \%)$ & $9.871(17,1 \%)$ \\
07. Scienze agrarie e veterinarie & $336(5,5 \%)$ & $3.040(5,3 \%)$ \\
08. Ingegneria civile ed Architettura & $332(5,4 \%)$ & $3.623(6,3 \%)$ \\
09. Ingegneria industriale e dell'informazione & $636(10,4 \%)$ & $5.290(9,2 \%)$ \\
10. Scienze dell'antichità, filologico-letterarie e storico-artistiche & $641(10,4 \%)$ & $5.227(9,1 \%)$ \\
11. Scienze storiche, filosofiche, pedagogiche e psicologiche & $639(10,4 \%)$ & $4.689(8,1 \%)$ \\
12. Scienze giuridiche & $371(6,0 \%)$ & $4.875(8,5 \%)$ \\
13. Scienze economiche e statistiche & $580(9,4 \%)$ & $4.825(8,4 \%)$ \\
14. Scienze politiche e sociali & $300(4,9 \%)$ & $1.752(3,0 \%)$ \\
\hline Scienze matematiche e informatiche & $435(7,1 \%)$ & $3.174(5,5 \%)$ \\
Scienze naturali & $1.216(19,8 \%)$ & $2.257(19,5 \%)$ \\
Scienze applicate & $1.957(31,9 \%)$ & $2.931(37,9 \%)$ \\
Scienze umane, Arte e Spettacolo & $1.280(20,9 \%)$ & $1.056(17,2 \%)$ \\
Scienze sociali & $1.251(20,4 \%)$ & $4.980(19,9 \%)$ \\
\hline
\end{tabular}


Tabella 3. - Caratteristiche socio-demografiche degli atenei di afferenza $(N=6.139)$.

\section{Campione}

\begin{tabular}{ccc} 
Statale & $5.737(93,5 \%)$ & $54.257(93,3 \%)$ \\
Non STAtale & $402(6,5 \%)$ & $3.918(6,7 \%)$ \\
\hline Tradizionale & $5.761(93,8 \%)$ & $57.122(98,2 \%)$ \\
Telematico & $378(6,2 \%)$ & $1.053(1,8 \%)$ \\
\hline
\end{tabular}

Tabella 4. - Area geografica delle sedi universitarie e macro-aree regionali $(N=6.139)$.

\begin{tabular}{|c|c|c|}
\hline & CAMPIONE & Popolazione $(\mathrm{N}=58.175)$ \\
\hline Valle d'Aosta & $17(0,3 \%)$ & - \\
\hline Piemonte & $395(6,4 \%)$ & - \\
\hline Liguria & $168(2,7 \%)$ & - \\
\hline Lombardia & $939(15,3 \%)$ & - \\
\hline Trentino-Alto Adige & $84(1,4 \%)$ & - \\
\hline Veneto & $406(6,6 \%)$ & - \\
\hline Friuli-Venezia Giulia & $168(2,7 \%)$ & - \\
\hline Emilia-Romagna & $591(9,6 \%)$ & - \\
\hline Toscana & $495(8,1)$ & - \\
\hline Marche & $196(3,2 \%)$ & - \\
\hline Umbria & $123(2,0 \%)$ & - \\
\hline Lazio & $920(15,0 \%)$ & - \\
\hline Abruzzo & $146(2,4 \%)$ & - \\
\hline Molise & $39(0,6 \%)$ & - \\
\hline Campania & $420(6,8 \%)$ & - \\
\hline Basilicata & $32(0,5 \%)$ & - \\
\hline Puglia & $289(4,7 \%)$ & - \\
\hline Calabria & $130(2,1 \%)$ & - \\
\hline Sicilia & $428(7,0 \%)$ & - \\
\hline Sardegna & $153(2,5 \%)$ & - \\
\hline Nord & $2.768(45,1 \%)$ & $24.744(42,5 \%)$ \\
\hline Centro & $1.734(28,2 \%)$ & $15.205(26,1 \%)$ \\
\hline Sud & $1.056(17,2 \%)$ & $11.932(20,5 \%)$ \\
\hline Isole & $581(9,5 \%)$ & $6.294(10,8 \%)$ \\
\hline
\end{tabular}


Un altro confronto è stato operato per quanto riguarda le caratteristiche degli atenei di afferenza. La comparazione delle due distribuzioni sulla variabile ateneo statale o ateneo non statale e quella ateneo tradizionale o ateneo telematico hanno evidenziato che le due distribuzioni sono simili per quanto attiene alla prima variabile $\left(\mathrm{X}^{2}(1)=0,340, p=, 056, r=, 01\right)$, mentre il confronto sulla variabile relativa alla tipologia di ateneo tradizionale o telematico ha evidenziato una sovrarappresentazione dei docenti degli atenei telematici $\left(\mathrm{X}^{2}(1)=652,796, p<, 01, r=, 37\right)$. Una possibile spiegazione può essere attribuibile al fatto che i docenti delle università telematiche fossero più motivati a partecipare all'indagine dal momento che hanno maggiore familiarità con le tecnologie di comunicazione a distanza (Tabella 3).

Infine, è stata presa in esame la variabile relativa alla distribuzione geografica. In questo caso è stato possibile operare il confronto solo in relazione alle macro-regioni. Poiché alcune università hanno più sedi, non si è potuto infatti attribuire la regione a partire dagli elenchi del MIUR che non contengono l'indicazione di appartenenza ad una specifica sede. Il risultato del confronto ha evidenziato (Tabella 4) che il campione e la popolazione presentano caratteristiche simili rispetto alla variabile geografica $\left(\mathrm{X}^{2}(3)=\right.$ $63,061, p<, 01, r=, 10)$.

\subsection{Diffusione dell'e-learning}

Seppur non specificamente orientato ad indagare la diffusione dell'e-learning, il questionario conteneva anche alcune domande mirate a rilevarne le pratiche d'uso. Nello specifico si è chiesto di indicare quali tipologie di insegnamento (in presenza, online e blended) fossero state erogate nell'ultimo anno accademico. Le risposte, illustrate nella Tabella 5, evidenziano una prevalenza massiccia di corsi tradizionali in presenza nelle tre casistiche di corsi.

Tabella 5. - Tipologie di insegnamenti tenuti nell'ultimo anno accademico $(N=5.997)$.

\begin{tabular}{cccc}
\hline & $\begin{array}{c}\text { INSEGNAMENTO } \\
\text { IN PRESENZA }\end{array}$ & $\begin{array}{c}\text { INSEGNAMENTO } \\
\text { ONLINE }\end{array}$ & $\begin{array}{c}\text { INSEGNAMENTO } \\
\text { BLENDED }\end{array}$ \\
\hline $\begin{array}{c}\text { Laurea triennale } \\
\text { Laurea magistrale, }\end{array}$ & $4.614(76,9 \%)$ & $173(2,9 \%)$ & $98(1,6 \%)$ \\
$\begin{array}{c}\text { dottorato, corsi post lauream } \\
\text { Altro (es. corsi }\end{array}$ & $4.789(79,9 \%)$ & $112(1,9 \%)$ & $122(2,0 \%)$ \\
di aggiornamento professionale) & $1.355(22,6 \%)$ & $63(1,1 \%)$ & $87(1,5 \%)$ \\
\hline
\end{tabular}


Tabella 6. - Tipologie di insegnamenti offerti dagli atenei $(N=3.674)$.

\begin{tabular}{cccc}
\hline & $\begin{array}{c}\text { NeSSUN } \\
\text { INSEGNAMENTO }\end{array}$ & $\begin{array}{c}\text { Solo SINGOLI } \\
\text { INSEGNAMENTI }\end{array}$ & $\begin{array}{c}\text { INSEGNAMENTI, } \\
\text { CORSI DI LAUREA, } \\
\text { CORSI POST LAUREAM }\end{array}$ \\
\hline ONLINE & $1.783(48,5 \%)$ & $746(20,3 \%)$ & $929(25,3 \%)$ \\
BLENDED & $1.366(37,2 \%)$ & $565(15,4 \%)$ & $439(11,9 \%)$ \\
\hline
\end{tabular}

Questo risultato può essere utilmente messo a confronto con quello della domanda successiva, che chiedeva di indicare le tipologie di corsi offerti dall'università di appartenenza. Come si può vedere nella Tabella 6 , secondo le risposte fornite quasi la metà degli atenei italiani $(48,5 \%)$ non offre alcun insegnamento online; questa percentuale si riduce, seppur di poco, se si fa riferimento alla modalità blended (37,2\%). Questo dato va preso con molta cautela, dal momento che andrebbe verificato con dati forniti da terze parti che abbiano fatto una ricognizione sistematica dell'offerta didattica delle università italiane.

Un'altra domanda chiedeva di indicare se si fosse mai fatto uso della piattaforma di e-learning (es. Blackboard, Moodle, WebCT, ecc.) della propria istituzione nell'ambito dei corsi e, in caso affermativo, in quali modi fosse stata utilizzata. Su 5.752 risposte, il 34,6\% dichiara di aver usato la piattaforma e-learning del proprio ateneo, contro il $58,0 \%$ che non l'ha mai usata. Un restante $7,4 \%$ dichiara che la sua università non dispone di una piattaforma di e-elearning.

Chi l'ha usata se ne è avvalso principalmente per inserire il programma dei corsi $(87,6 \%)$, per inviare messaggi o avvisi agli studenti $(72,0 \%)$, per consultare l'archivio delle risorse multimediali e dei contenuti digitali $(50,4 \%)$ e per gestire i compiti $(46,0 \%)$ e i voti $(42,2 \%)$ degli studenti. Infine, il $42,1 \%$ dichiara di aver usato anche i forum di discussione. Nei commenti a questa domanda emerge che l'uso prevalente riguarda l'erogazione dei contenuti (ad es. fornire materiale didattico, rendere disponibili le slide della lezione, caricare registrazioni audio o video delle lezioni), piuttosto che la creazione di gruppi di lavoro per attività di tipo collaborativo. Infatti, solo molto raramente si accenna ad attività orientate allo sviluppo di comunità di apprendimento.

E stato, inoltre, chiesto quali funzionalità generalmente non presenti in una piattaforma di e-learning fossero maggiormente desiderabili. In una Scala Likert a 4 punti da Non importante a Molto importante, i valori più alti (Molto importante e Importante) sono stati attribuiti all'accesso alle risorse bibliotecarie della propria università $(75,2 \%)$, l'accesso a risorse open source 
$(73,6 \%)$ e l'accesso a materiali messi a disposizione dall'editoria accademica $(65,6 \%)$. Minore interesse sembrano, invece, rivestire il collegamento con Social Media esterni $(36,6 \%)$ e la presenza di funzioni di supporto alla socializzazione all'interno della piattaforma $(31,1 \%)$.

\subsection{Social Media e didattica}

La relazione tra Social Media e loro utilizzo in ambito didattico è stata investigata in una serie di domande, a partire dalla frequenza d'uso di questi strumenti, che è stata autovalutata secondo una Scala Likert a 5 punti, che andava da Mai a Almeno una volta al giorno. Il 36,0\% del campione ha dichiarato di non usare alcuno strumento. Questa percentuale sale al 55,1\% se si considera l'uso almeno mensile. Nel dettaglio la situazione, riferita al campione dei 6.139 rispondenti, è quella illustrata nella Tabella 7 , da cui si evince come i Social Media più usati siano YouTube e Vimeo e blog e wiki.

La percezione di utilità che questi strumenti rivestono per la didattica è stata indagata attraverso la domanda che chiedeva «Ritieni che i Social Media ti siano utili nella didattica?» $(\mathrm{N}=5.833)$. A parte una quota consistente di chi esprime indecisione $(24,1 \%)$, il restante delle risposte si distribuisce quasi equamente tra coloro che ritengono che i Social Media siano utili nella didattica $(38,6 \%)$ e coloro che al contrario ritengono che non lo siano $(37,4 \%)$.

Tabella 7. - Frequenza d'uso didattico dei diversi strumenti $(N=6.139)$.

\begin{tabular}{|c|c|c|c|c|c|}
\hline & $\begin{array}{l}\text { ALMENO } \\
\text { UNA VOLTA } \\
\text { AL GIORNO }\end{array}$ & $\begin{array}{c}\text { AlMENO } \\
\text { UNA VOLTA } \\
\text { ALLA SETTIMANA }\end{array}$ & $\begin{array}{l}\text { Almeno } \\
\text { UNA VOLTA } \\
\text { AL MESE }\end{array}$ & RARAMENTE & MAI \\
\hline Twitter & $0,5 \%$ & $1,0 \%$ & $1,0 \%$ & $2,9 \%$ & $94,5 \%$ \\
\hline Facebook & $2,7 \%$ & $4,8 \%$ & $3,6 \%$ & $7,0 \%$ & $82,0 \%$ \\
\hline LinkedIn & $0,5 \%$ & $1,5 \%$ & $1,9 \%$ & $5,6 \%$ & $90,5 \%$ \\
\hline Podcast & $0,5 \%$ & $1,2 \%$ & $1,9 \%$ & $5,2 \%$ & $91,2 \%$ \\
\hline Blog e wiki & $3,0 \%$ & $8,2 \%$ & $7,7 \%$ & $9,8 \%$ & $71,3 \%$ \\
\hline $\begin{array}{l}\text { YouTube } \\
\text { e Vimeo }\end{array}$ & $1,9 \%$ & $7,8 \%$ & $11,4 \%$ & $18,1 \%$ & $60,7 \%$ \\
\hline $\begin{array}{l}\text { ReearchGate } \\
\text { e Academia.edu }\end{array}$ & $2,5 \%$ & $6,0 \%$ & $6,4 \%$ & $10,8 \%$ & $74,4 \%$ \\
\hline SlideShare & 1,0 & $3,0 \%$ & $4,0 \%$ & $7,5 \%$ & $84,5 \%$ \\
\hline
\end{tabular}


Tra le ragioni espresse a favore nei commenti troviamo alcuni dei motivi indicati anche nel questionario rispetto ai singoli strumenti (che vedremo sotto). Alcuni sottolineano come l'impiego di questi dispositivi migliori la motivazione e accresca il coinvolgimento degli studenti, mentre in diversi insistono sull'opportunità che i Social Media offrono di realizzare forme di didattica collaborativa e partecipativa. Ulteriori motivazioni che ricorrono nei commenti liberi riguardano il fatto che questi strumenti permettono di condividere in modo più facile e immediato materiali e informazioni, consentendo di offrire risorse sempre aggiornate e materiali integrativi rispetto alla lezione. Significativo è anche il ruolo assegnato ai Social Media come strumenti per comunicare con gli studenti e rimanere in contatto con loro.

Chi, invece, ha espresso una posizione negativa adduce motivazioni quali l'indebolimento dei ruoli tradizionali di studente e docente, gli effetti di distrazione sugli studenti, l'eccessiva perdita di tempo, le problematiche legate alla privacy, la perdita del calore dei contatti reali, l'insuperabile efficacia della tradizionale lezione frontale. Qualcuno evidenzia come gli studenti non siano affatto interessati ad utilizzare questi strumenti per attività di studio, oppure sottolinea come l'impiego di social network alla Facebook possa condurre ad un globale impoverimento culturale degli studenti o ad un appiattimento delle pratiche accademiche. In generale, ricorre con una certa frequenza l'osservazione secondo cui, essendo il proprio ateneo dotato di piattaforma di e-learning, non è necessario avvalersi di altri strumenti. In particolare, per alcuni questa piattaforma consente di gestire la comunicazione con gli studenti e la condivisione dei materiali in modo più efficace di altri strumenti, il cui uso frammentario potrebbe risultare dispersivo. Per altri la piattaforma di ateneo consente maggiori livelli di protezione e sicurezza, caratteristica che li renderebbe preferibili ai Social Media.

Ad una domanda successiva, rivolta solo a coloro che avevano risposto affermativamente alla domanda precedente $(\mathrm{N}=2.249)$, che chiedeva di indicare i Social Media ritenuti più utili, le risposte indicano al primo posto YouTube e Vimeo $(53,5 \%)$ perché ricchi di materiali integrativi per la didattica: da documentari utili per l'insegnamento delle scienze a filmati didattici esemplificativi di aspetti anche pratici delle discipline oggetto di studio, a contenuti in lingua originale per l'insegnamento delle lingue straniere. I due Social Media sono seguiti dai blog e wiki $(37,8 \%)$ e Facebook (34,6\%). Per un resoconto completo, si veda la Figura 1.

Una domanda ulteriore si rivolgeva a chi avesse usato questi strumenti chiedendo quale fosse la motivazione principale. Le risposte $(332<\mathrm{N}<1.577)$ hanno evidenziato una situazione piuttosto variegata. 


\section{Social Media ritenuti più utili nella didattica (\%)}

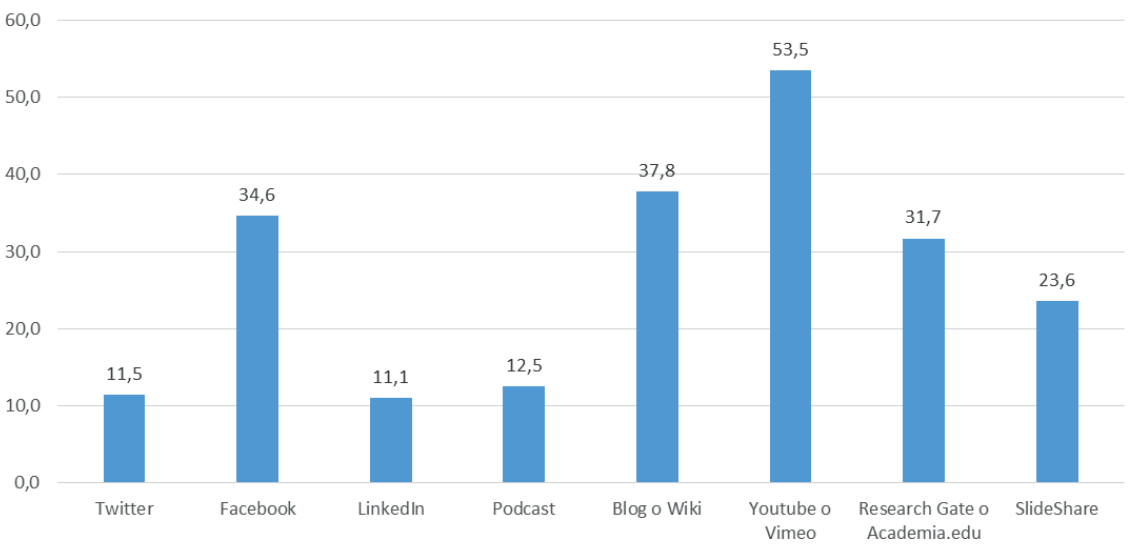

Figura 1. - I Social Media ritenuti più utili da un punto di vista didattico $(N=2.249)$.

Come illustrato nella Figura 2, gli strumenti considerati più motivanti e coinvolgenti per gli studenti sono stati Facebook (25,5\% su 911 risposte), Twitter (25,3\% su 332 risposte) e YouTube e Vimeo (25,0\% su 1.577). Come supporto per realizzare forme di didattica collaborativa e partecipativa, $\mathrm{i}$ blog e wiki hanno ottenuto il punteggio più alto (22,3\% su 1.049 risposte), seguiti da Facebook (20,1\% su 911 risposte) e LinkedIn (19,5\% su 329 risposte); per la possibilità di condividere facilmente materiali didattici con gli studenti gli strumenti più usati sono stati SlideShare (39,4\% su 599 risposte) e ResearchGate e Academia.edu (28,3\% su 826 risposte); mentre come strumento per migliorare la qualità della didattica in generale sono stati usati più frequentemente SlideShare (31,9\% su 599 risposte), seguito da YouTube e Vimeo (28,5\% su 1.577$)$ e dai podcast (27,7\% su 358 risposte). Infine, la motivazione basata sulla diffusa familiarità presso gli studenti ha inciso, seppur in misura contenuta, nella scelta di Facebook (22,5\% su 911 risposte) e Twitter (14,2\% su 332 risposte); mentre la possibilità di sperimentare nuovi strumenti per la didattica ha costituito pressoché in tutti i casi una motivazione molto bassa, sempre al di sotto del $15 \%$ delle risposte.

Altre motivazioni e modalità di utilizzo sono state indagate nelle risposte aperte a questa domanda. Queste hanno evidenziato che Twitter e Facebook vengono anche utilizzati per motivi pratici, ad esempio il primo come strumento per comunicazioni rapide con gli studenti (avvisi su esami, lezioni, ricevimenti, orari, eventi), mentre il secondo per la gestione del calendario. 
옹

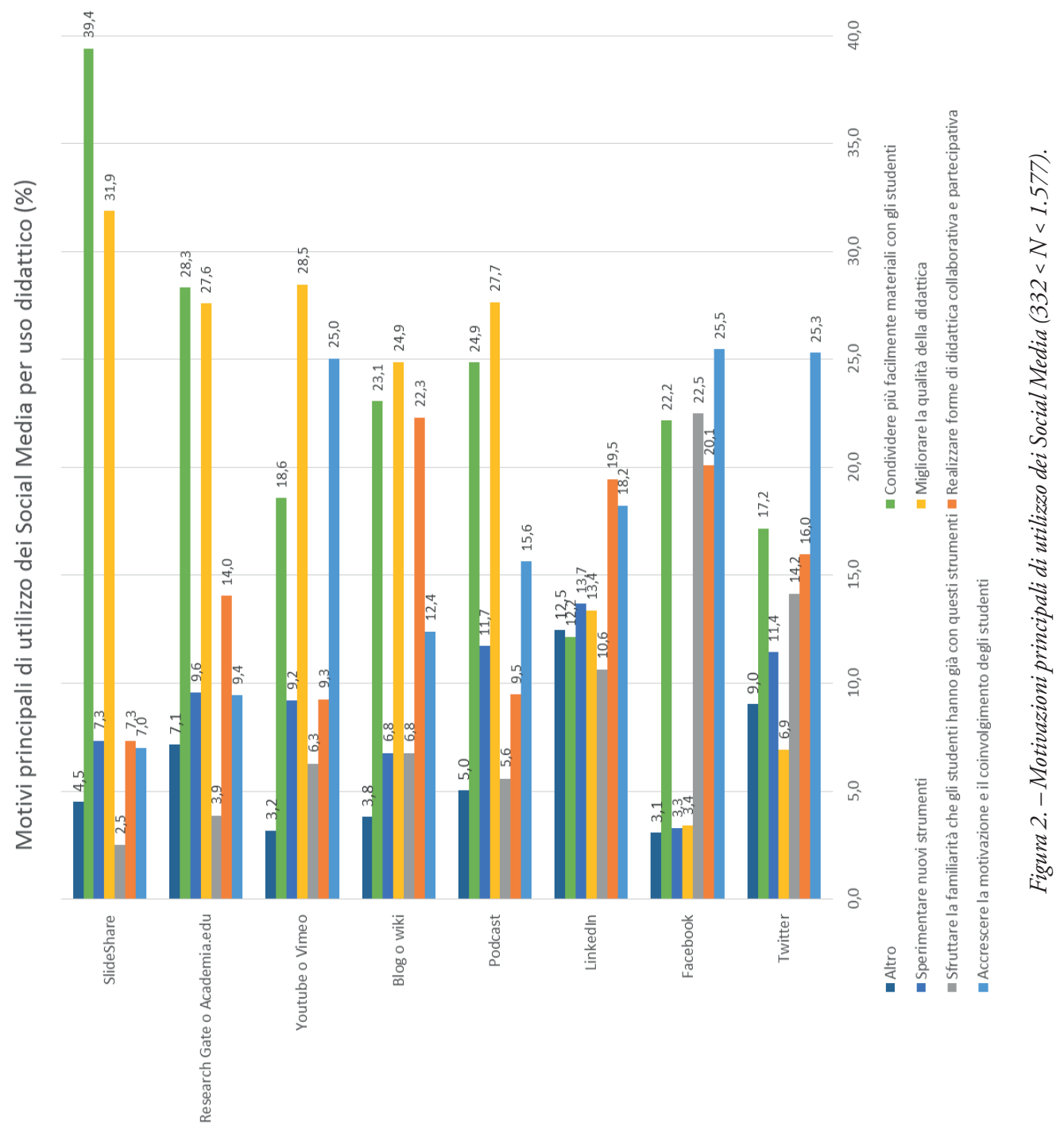


Facebook insieme a blog e wiki vengono particolarmente apprezzati per creare gruppi di lavoro, anche informali, mentre i podcast per l'ascolto di lezioni registrate. I commenti su LinkedIn e SlideShare sono meno numerosi: il primo appare a prima vista non immediatamente spendibile sul piano didattico, il secondo sembra essere meno noto. Tuttavia, i commenti su LinkedIn evidenziano soprattutto la possibilità di mantenere i contatti con studenti e laureati e le implicazioni per il job placement, mentre SlideShare viene menzionato come utile per preparare le lezioni. Infine, ResearchGate e Academia.edu vengono indicati come ambienti funzionali al reperimento di materiale bibliografico e alla creazione di reti su scala internazionale.

Strettamente legata a quella sulle motivazioni è la domanda finalizzata a sondare che tipo di usi i docenti hanno fatto relativamente ai diversi strumenti considerati $(233<\mathrm{N}<1.829)$. Tra le opzioni a risposta chiusa i rispondenti avevano a disposizione "Gli studenti dovevano visionare materiali», «Gli studenti dovevano commentare materiali», «Gli studenti dovevano produrre elaborati» e "Altra modalità». I risultati, illustrati nella Figura 3, evidenziano come la visione di materiali già predisposti sia stata privilegiata in ambienti quali YouTube e Vimeo (69,6\% su 1.829 risposte), SlideShare (64,4\% su 581 risposte) e ResearchGate e Academia.edu (58,8\% su 706 risposte), il commento di materiali abbia prevalso su Facebook (24,2\% su 939 risposte) e Twitter (23,1\% su 316 risposte), mentre la produzione di nuovi materiali o elaborati sia stata affidata principalmente ai blog e wiki $(21,4 \%$ su 1.203). I commenti alle risposte chiuse confermano sostanzialmente queste tipologie di utilizzo con qualche precisazione. Alcuni enfatizzano la caratteristica di questi ambienti di fornire molteplici fonti informative e di varia natura (esperti, documenti, siti, ecc.), alcune delle quali possono essere raccolte, archiviate e mostrate a lezione; altri, invece, ne sottolineano la valenza dialogico-comunicativa nella misura in cui sono in grado di supportare discussioni e scambi che si estendono oltre la lezione.

Un'altra domanda mirava a sondare l'efficacia dei Social Media come strumenti per la costruzione di una comunità di studenti/ex studenti. Su 5.789 risposte totalizzate, il 65,2\% ritiene che possano essere efficaci, contro l' $11,2 \%$, mentre il $23,6 \%$ non ha una posizione né a favore né contraria. Tra le ragioni favorevoli vengono annoverate motivazioni quali la facilitazione degli scambi, il superamento delle distanze, il supporto per i non frequentanti, la possibilità di mantenere attivi legami personali e favorire la conoscenza reciproca delle esperienze professionali per future e/o inedite collaborazioni, la creazione di reti di laureati inseriti nel mondo del lavoro in grado di fornire consigli utili a chi ancora studia, l'aumento della fidelizzazione. È interessante notare come dai commenti emergano due visioni molto diverse sul modo in cui tali comunità dovrebbero essere organizzate. 
:

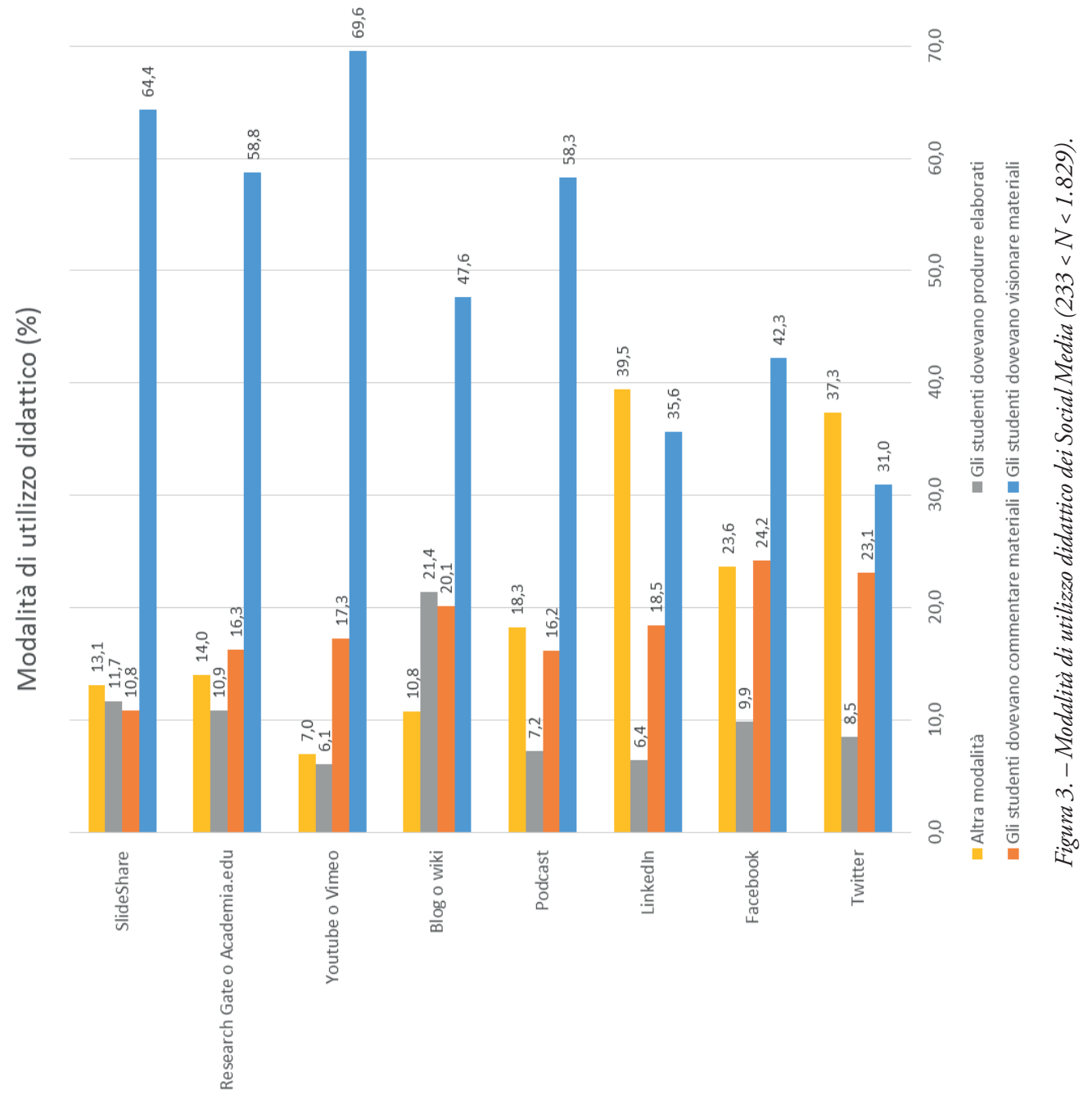


Da un lato, prevale l'idea secondo cui simili comunità dovrebbero essere gestite interamente dagli alunni o ex alunni senza alcun intervento dei docenti: ambienti come Facebook, del resto, sono nati come strumenti informali per ritrovare vecchi compagni di scuola, generando comunità spontanee di ex alunni. Dall'altro, c'è chi sostiene che la nascita di comunità di questo tipo dovrebbe essere inquadrata nell'alveo delle iniziative istituzionali e regolata di conseguenza sulla base di precise policy pubbliche. I contrari argomentano perlopiù che si tratta di strumenti dispersivi e inutili che favoriscono solo lo sviluppo di relazioni superficiali, mentre le comunità andrebbero favorite attraverso contatti diretti. Gli indecisi, infine, commentano che la loro efficacia dipende dalla finalità e dal modo in cui sono utilizzati: da un lato, si registrano indubbi vantaggi, ad esempio di reperimento di informazioni, anche difficili da recuperare, dall'altro, rischi di distrazione con conseguente perdita di tempo e ricadute negative sullo studio. Anche tra gli indecisi alcuni sottolineano la necessità di ricondurre tali comunità al contesto istituzionale, avvalendosi di strumenti formalmente riconosciuti dagli atenei, come ad esempio le piattaforme di e-learning, meno dispersive e pertanto più efficaci.

Infine, è stato chiesto quali ostacoli possono impedire l'uso didattico dei Social Media $(2.960<\mathrm{N}<3.760)$. Tra le ragioni di impedimento al primo posto risultano i problemi relativi all'accertamento dell'autenticità dei contributi prodotti dagli studenti $(83,2 \%$ su 3.760 risposte), la mancanza di funzionalità specifiche per la didattica $(74,8 \%$ su 3.359$)$ e la carenza di buone pratiche per alcuni strumenti (73,3\% su 3.110 risposte). Ulteriori ragioni sono state individuate nella dispersione dei partecipanti e delle informazioni nei diversi ambienti $(69,1 \%$ su 3.157 risposte) e nella difficoltà di valutare le attività didattiche svolte $(68,2 \%$ su 3.250 risposte) . Per una rappresentazione completa delle risposte a questa domanda si veda la Figura 4.

Tra i commenti sono stati evidenziati ulteriori fattori di criticità quali mancanza di tempo, assenza di strutture e supporto organizzativo, mancato riconoscimento del carico di lavoro aggiuntivo sia per gli studenti che per i docenti, scarso interesse degli studenti ad utilizzare queste modalità comunicative e di lavoro a scopo accademico, necessità di tenere distinte identità personale e identità professionale, assenza di politiche sul copyright. A monte, però, emerge come questione di fondo la seguente: in molti non ritengono che i Social Media siano funzionali alla didattica o, meglio, alla propria specifica disciplina di insegnamento; alcuni evidenziano addirittura come essi siano intrinsecamente diseducativi. In questo caso, tuttavia, più che parlare di ostacoli all'adozione, appare corretto ricondurre questi commenti alla categoria delle ragioni che spingono a non utilizzare i Social Media. 


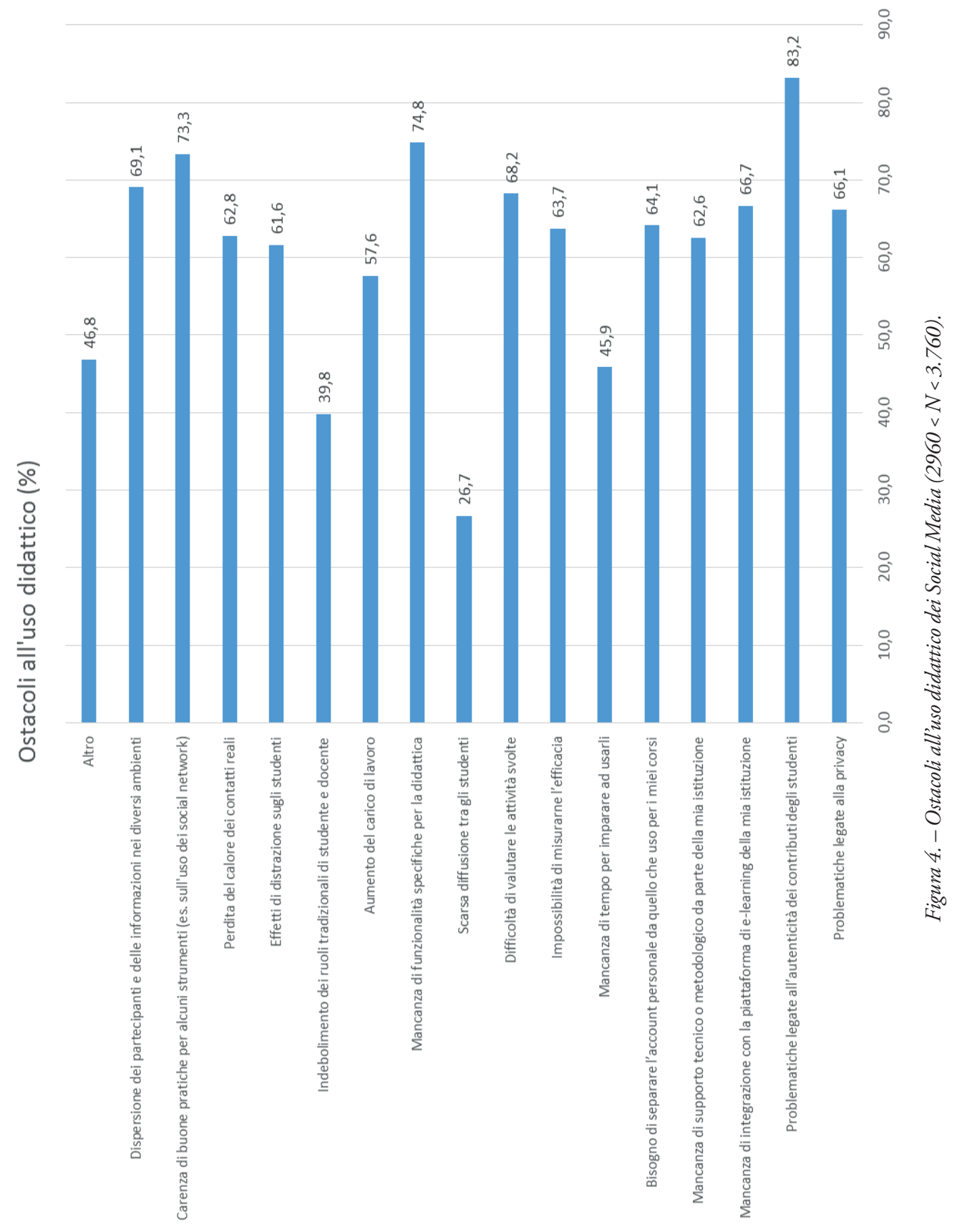


Tra queste ricorre frequentemente, qui come già prima (vedi commenti sui motivi di uso o non uso), anche la mancanza del contatto diretto: la comunicazione faccia-a-faccia come medium privilegiato della lezione tradizionale rimane per molti docenti irrinunciabile in quanto strumento essenziale per garantire la qualità dei rapporti con gli studenti e della didattica. Viene ribadita, inoltre, l'inaffidabilità dei servizi social a fronte delle piattaforme e-learning istituzionali.

Infine, dai commenti finali al questionario emergono due principali tendenze relative alle reazioni dei rispondenti. Gran parte delle osservazioni riguardano apprezzamenti positivi verso l'area d'investigazione della presente indagine: l'attualità dell'argomento, la diffusione dei Social Media tra le nuove generazioni, la mancanza di ricerche e riflessioni su questi temi e le potenzialità percepite per la didattica sono le ragioni tipicamente addotte per argomentare a favore della positività dell'indagine. Al tempo stesso, numerosi sono i commenti in cui si ribadiscono posizioni di profondo scetticismo o di vera e propria ostilità verso l'uso dei Social Media nelle attività accademiche. Dispersività, ridondanza, distrazione, perdita o mancanza di tempo, assenza di supporto istituzionale, mancanza di funzionalità didattiche specifiche e gap generazionale si confermano come fattori che generano scarso interesse verso l'adozione dei Social Media nei contesti universitari.

\section{Discussione}

I risultati indicano che, anche se ben oltre la maggioranza degli accademici ha fatto uso di almeno un Social Media nella propria attività didattica, solo un terzo ne vede l'utilità, soprattutto per quanto riguarda l'uso di applicazioni come YouTube e Vimeo. La dimensione erogativa, inoltre, sembra prevalere su quella dialogico-interattiva: le piattaforme e-learning d'ateneo vengono utilizzate più per erogare programmi e lezioni che per interagire. Questo dato è in linea con la netta preferenza accordata a strumenti per la visualizzazione di materiali come YouTube, Vimeo e SlideShare, piuttosto che a strumenti per condividere e interagire come Facebook e Twitter o per produrre come blog e wiki. Nonostante solo un terzo dei rispondenti ritenga che i Social Media siano utili nella didattica, ben oltre la maggioranza li ritiene efficaci per la costruzione di comunità di studenti o ex studenti; non mancano, tuttavia, molteplici e ricorrenti richiami nei commenti liberi all'uso delle piattaforme d'ateneo, percepite come strumenti più efficaci per la gestione della comunicazione e meglio controllabili. Infine, tra i maggiori ostacoli all'uso dei Social Media nella didattica vengono annoverati il problema dell'autenticità 
dei contributi prodotti dagli studenti, la mancanza di funzionalità specifiche e la carenza di buone pratiche.

Questi risultati non possono essere letti in modo univoco e non per eventuali limitazioni dello studio (si veda sotto), ma perché riflettono una ambivalenza presente anche in letteratura rispetto alle potenzialità o meno dei Social Media per l'alta formazione.

Un primo elemento sul quale appare rilevante riflettere riguarda la percezione di inutilità. Si tratta a questo proposito di comprendere fino a che punto agiscano, all'origine di tale percezione, resistenze riconducibili alle pedagogie implicite che alimentano le pratiche didattiche degli accademici e fino a che punto intervengano problematiche di carattere pragmatico. Dai risultati e dai commenti le due prospettive appaiono convivere. Da un lato, la preferenza accordata a dispositivi per l'erogazione e la visualizzazione dei contenuti insieme all'insistenza sull'efficacia della lezione frontale e della comunicazione vis- $\grave{a}$-vis rinviano ad un preciso modo di intendere la relazione pedagogica, sostanzialmente caratterizzata dalla trasmissione unilaterale dei contenuti. Chi frequenta le aule universitarie sa che spesso questo è un formato didattico imposto dalle condizioni logistiche in cui il docente si trova ad operare. Tuttavia, l'orientamento prevalente legato alla trasmissione del contenuto, che evidenzia una visione decisamente poco compatibile con l'uso dei Social Media, sembra indicare soprattutto quella resistenza al cambiamento di cui parlano, ad esempio, Collis e Moonen (2008). Dall'altro, sembrano agire fattori di natura pragmatica che ricorrono anche in altri studi come quelli di Brown (2012) e di Conole (2013): per alcuni docenti, determinati contenuti disciplinari non si prestano ad essere spiegati e compresi attraverso i Social Media; oppure i Social Media risultano troppo dispersivi rischiando di rendere inefficace l'accesso alle risorse e all'informazione; o, ancora, esistono strumenti più pratici, come le piattaforme d'ateneo, che non giustificano l'utilizzo di ulteriori strumenti. In breve, «la ragion pratica» sembrerebbe avere la meglio sul fascino del nuovo.

Il richiamo all'uso della piattaforma d'ateneo, più volte evidenziato in questo studio, merita però un approfondimento ulteriore: perché tale richiamo è così ricorrente? Oltre agli aspetti pedagogico-didattici (piattaforma intesa come strumento di erogazione dei contenuti didattici) e a quelli pragmatici sopra riportati, sembrano intervenire anche fattori di natura valoriale in linea con quanto emerso dallo studio di Veletsianos e Kimmons (2013): le piattaforme consentono di controllare meglio lo spazio della comunicazione, garantendo la sicurezza degli scambi dialogici, limitando i rischi per la privacy e contenendo l'erosione dei confini tra identità professionale e personale. I Social Media allo stato attuale non permettono di gestire in modo efficace questi aspetti, con implicazioni negative anche rispetto alla gestione dei tempi: laddove il profes- 
sionale sconfina nel personale, e viceversa, si evidenzia un deciso aumento del carico di lavoro per il docente che si troverebbe a gestire un traffico di comunicazione elettronica moltiplicato. La propensione all'impiego delle piattaforme sembra giustificata anche dal maggiore controllo che esse consentono di esercitare sulla valutazione dei compiti degli studenti e sulla loro autenticità. Emerge qui una delle possibili fonti di tensione richiamate da Crook (2008): come valutare contributi oggetto di produzione collaborativa e sociale in un contesto in cui la valutazione rimane una questione sostanzialmente individuale? In realtà, l'idea secondo cui l'utilizzo delle piattaforme garantisca un maggiore controllo sull'autenticità dei contributi e sulla imputabilità degli stessi rischia di diventare un mito, tenendo conto del fatto che dietro un qualsiasi schermo può anche esservi un cane, tanto per citare un famoso adagio. La fiducia si costruisce attraverso altri canali; tuttavia sul piano delle percezioni diffuse le piattaforme comunicano controllo e sicurezza su più fronti.

Cosa ci si aspetta dagli studenti? Per quanto nessuna domanda indagasse in modo diretto la rappresentazione che gli accademici hanno delle nuove generazioni di studenti, nei commenti non sono mancati riferimenti ai loro principali interlocutori. In generale, sono visti come utilizzatori attivi di queste nuove tecnologie. Solitamente a questa rappresentazione si abbinano considerazioni negative alla Carr (2010): non solo i Social Media non sono utili nella didattica universitaria, ma il loro uso intensivo da parte degli studenti provoca gravi conseguenze negative sul piano culturale, soprattutto l'impoverimento delle capacità cognitive e la perdita di tempo prezioso sottratto allo studio. Alla base di questa idea sembra anche esservi una rappresentazione decisamente negativa dei Social Media in se stessi, al di là dell'uso - positivo o negativo - che ne viene fatto. In più di un commento vengono ascritti ai Social Media attributi negativi come se essi fossero dotati di qualità intrinseche, astoriche e indipendenti dagli specifici contesti culturali e sociali di utilizzo. Si tratta di una visione sostanzialmente tecnofobica, di marca deterministica tanto quanto le visioni dei tecnoentusiasti. Ma, come argomentato altrove (Ranieri, 2011), queste posizioni non aiutano ad articolare il rapporto tra tecnologie ed educazione al di fuori delle visioni stereotipate.

Voci fuori dal coro sono quelle di docenti che hanno evidenziato come gli studenti non siano contenti di utilizzare i Social Media per attività di studio: qualora sia stato proposto, ciò non è stato accolto come un invito allettante a studiare diversamente. Riteniamo, tuttavia, che quest'area di ricerca andrebbe ulteriormente esplorata. L'adozione efficace delle tecnologie nella didattica non dipende, infatti, solo dalle credenze dei docenti, ma anche dalle percezioni degli studenti. Esistono alcuni studi a riguardo (cfr. Selwyn, 2009) che confermano un atteggiamento sostanzialmente poco favorevole, ma tali ricerche non sono ancora molte. 


\section{LIMITAZIONI DELLO STUDIO}

Seppure la ricerca sia riuscita nell'intento di rivolgersi a tutta la popolazione di ricerca universitaria italiana, lo studio presenta una serie di limitazioni importanti. La principale riguarda il tasso di risposta che, come abbiamo visto, è stato del $10,5 \%$. Sebbene le indagini online ottengano un tasso di risposta mediamente inferiore dell' $11 \%$ rispetto a somministrazioni tradizionali, quali i questionari cartacei o le indagine telefoniche (Fan \& Yan, 2010), nel nostro caso ci sono state una serie di difficoltà oggettive relative al reperimento degli indirizzi e-mail o all'effettiva ricezione delle mail inviate. Non tutti gli indirizzi e-mail presenti nei siti web delle università erano corretti e non sempre quelli generati secondo l'algoritmo individuato sono correttamente arrivati a destinazione. Inoltre, con l'uso sempre più diffuso di tecniche di filtraggio di mail indesiderate, le mail di invito possono essere state bloccate dai filtri anti-spam. A ciò si aggiunga che non tutti i docenti universitari consultano la loro mail istituzionale, preferendo quella personale. Inoltre, nonostante la diffusione degli strumenti digitali e l'accesso ad Internet possano essere dati per acquisiti specie in una popolazione come quella accademica, si evidenziano ancora delle differenze di familiarità e di frequenza di accesso a strumenti di compilazione online in alcune fasce della popolazione (Teo, 2013), oltre a caratteristiche socio-demografiche che possono predisporre o inibire la volontà di partecipare ad una survey online (Diment \& Garrett-Jones, 2007).

Ulteriori motivi per un tasso di risposte così basso possono essere ascrivibili alla mancanza di familiarità con il tema, all'opposizione pregiudiziale nei confronti dell'argomento e al tempo richiesto per la compilazione (20 minuti).

Infine, dal momento che l'indagine richiedeva ai partecipanti di avere esperienza di insegnamento, questo requisito può aver fatto desistere dalla compilazione una quota di quel $46,6 \%$ costituito da ricercatori che non ricoprono incarichi di insegnamento.

\section{ConClusioni}

Questo lavoro ha inteso indagare le pratiche didattiche degli accademici italiani, con particolare riferimento all'uso dei Social Media. Nonostante le sue limitazioni, da esso emerge come i Social Media rimangano per ora ai margini della vita accademica in Italia. Una combinazione di fattori di natura pedagogica, pragmatica e valoriale sembrano indurre i docenti a preferire la didattica tradizionale in presenza o al più le tradizionali piattaforme e-learning. A ciò 
si aggiunga anche una percezione sulle effettive aspettative degli studenti ancora incerta, talora accompagnata da una rappresentazione dei Social Media deterministicamente orientata in senso negativo.

Tali elementi, presi insieme, sollevano molteplici questioni. In particolare, a monte ci si potrebbe chiedere se, a fronte di tanta diffidenza, abbia un senso o un valore utilizzare i Social Media per la didattica universitaria, e più in generale per l'apprendimento. Piaccia o non piaccia, i Social Media mediano oggi in forme sempre più massicce l'accesso, la condivisione e la produzione di informazioni. Tutto questo non può non avere rilevanza per l'alta formazione. Così, piuttosto che ragionare in termini deterministici, crediamo che la vera sfida sia quella di capire meglio quali siano le pratiche diffuse, le percezioni correnti e i principali ostacoli nell'ottica di poter formulare indicazioni utili che possano supportare il docente nella scelta delle soluzioni tecnologico-didattiche più funzionali.

Attraverso questo studio abbiamo tentato di produrre un avanzamento in tal senso, offrendo un contributo agli studi condotti nel quadro del più ampio fenomeno della digital scholarship (Weller, 2011; Greenhow \& Gleason, 2014). Con questa espressione ci si riferisce alle diverse pratiche che caratterizzano la vita professionale degli accademici (dalla ricerca alla didattica) realizzate attraverso l'utilizzo delle tecnologie digitali. Si tratta di un ambito rispetto al quale la letteratura è ancora agli inizi, specie per quanto riguarda il nostro paese, ma che merita sicuramente di essere indagato per impostare politiche sul sistema dell'alta formazione maggiormente informate.

\section{RINGRAZIAMENTI}

Si ringraziano Donatella Persico e Cinzia Cervato per aver contribuito alla traduzione e adattamento del testo dell'indagine. Un ringraziamento speciale a Carlo Chiorri per la consulenza statistica. Un sentito ringraziamento anche a tutti i colleghi universitari che hanno partecipato allo studio. 


\section{RIFERIMENTI BIBLIOGRAFICI}

Ajjan, H., \& Hartshorne, R. (2008). Investigating faculty decisions to adopt Web 2.0 technologies: Theory and empirical tests. The Internet and Higher Education, 11(2), 71-80.

Allen, I. E., \& Seaman, J. (2014). Grade change tracking. Online education in the United States. Babson Survey Research Group and Quahog Research Group, LLC.

Anderson, T. (2006). PLE's versus LMS: Are PLEs ready for Prime time? http://terrya. edublogs.org/2006/01/09/ples-versus-lms-are-ples-ready-for-prime-time/.

Anderson, T. (2009). Social networking. In S. Mishra (Ed.), Stride handbook 8 E-learning. Indira Gandhi National Open University.

Avci, U., \& Askar, P. (2012). The comparison of the opinions of the university students on the usage of blog and wiki for their courses. Educational Technology \& Society, 15(2), 194-205.

Bonaiuti, G. (a cura di). (2006). E-learning 2.0. Trento: Erickson.

Brown, J. S., \& Adler, R. P. (2008). Minds on fire: Open education, the long tail, and learning 2.0. Educause Review, 43(1), 16-32.

Brown, S. A. (2012). Seeing Web 2.0 in context: A study of academic perceptions. The Internet and Higher Education, 15(1), 50-57.

Caers, R., \& Castelyns, V. (2011). LinkedIn and Facebook in Belgium: The influences and biases of social network sites in recruitment and selection procedures. Social Science Computer Review, 29(4), 437-448.

Carr, N. (2010). Internet ci rende stupidi? Come la rete sta cambiando il nostro cervello. Milano: Raffaello Cortina.

Chiorri, C. (2014). Fondamenti di psicometria. Milano: McGraw-Hill.

Churchill, D. (2009). Educational applications of Web 2.0: Using blogs to support teaching and learning. British Journal of Educational Technology, 40(1), 179-183.

Churchill, D. (2011). Web 2.0 in education: A study of the explorative use of blogs with a postgraduate class. Innovations in Education and Teaching International, 48(2), 149-158.

Codina, L. (2009). Science 2.0: Social networks and online applications for scholars. Hipertext.net, 7.

Collis, B., \& Moonen, J. (2008). Web 2.0 tools and processes in higher education: Quality perspectives. Educational Media International, 45(2), 93-106.

Conole, G. (2013). Designing for learning in an open world (explorations in the learning sciences, instructional systems and performance technologies). Berlin: Springer.

Cress, U. (2012). Educational technology in Europe: Current issues from the learning sciences perspectives. Educational Technology, 52(2), 14-17.

Crook, Ch. (2008). Web 2.0 technologies for learning: The current landscape - Opportunities, challenges, and tensions. BECTA Report. 
Davis III, C. H. F., Deil-Amen, R., Rios-Aguila, C., \& Sacramento Gonzalez Canche, M. (2012). Social Media in higher education: A literature review and research directions. The Center for the Study of Higher Education at the University of Arizona - Claremont Graduate University.

de Waard, I., Abajian, S., Gallagher, M. S., Hogue, R., Keskin, N., Koutropoulos, A., \& Rodriguez, O. C. (2011). Using mLearning and MOOCs to understand chaos, emergence, and complexity in education. The International Review of Research in Open and Distance Learning, 12(5), 94-114.

Diment, K., \& Garrett-Jones, S. (2007). How demographic affect mode preference in a postal/web mixed-mode survey of Australian researchers. Social Science Computer Review, 25(3), 410-417.

Downes, S. (2005). E-learning 2.0. eLearn Magazine, October. http://elearnmag. acm.org/featured.cfm?aid=1104968.

Downes, S. (2010). Deinstitutionalizing education. Huffington Post, 2 November. http:// www.huffingtonpost.com/stephen-downes/deinstitutionalizing-educ_b_777132. html.

Dron, J., \& Anderson, T. (2009). Lost in social space: Information retrieval issues in Web 1.5. Journal of Digital Information, 10(2).

Esposito, A. (2011). Social Media e didattica universitaria: tensioni e casi studio nel panorama internazionale. In M. B. Ligorio, E. Mazzoni, A. Simone, \& M. Casini Schaerf, (a cura di), Didattica universitaria online: teorie, esperienze, strumenti. Napoli: ScriptaWeb.

Facebook (2014). Key Facts. https://newsroom.fb.com/key-Facts.

Fan, W., \& Yan, Z. (2010). Factors affecting response rates of the web survey: A systematic review. Computers in Human Behavior, 26(2), 132-139.

Fedeli, L. (2012). Social Media e didattica. Opportunità, criticità e prospettive. Lecce: Pensa MultiMedia.

Fini, A., \& Cigognini, E. (a cura di). (2009). Web 2.0 e social networking. Nuovi paradigmi per la formazione. Trento: Erickson.

Gao, F., Luo, T., \& Zhang, K. (2012). Tweeting for learning: A critical analysis of research on microblogging in education published in 2008-2011. British Journal of Educational Technology, 43(5), 783-801.

Gerard, J. G. (2012). Linking in with LinkedIn ${ }^{\oplus}$ : Three exercises that enhance professional social networking and career building. Journal of Management Education, 36(6), 866-897.

Greenhow, C., \& Gleason, B. (2014). Social scholarship: Reconsidering scholarly practices in the age of social media. British Journal of Educational Technology, 45(3), 392-402.

Greenhow, C., Gleason, B., \& Li, J. (2014). Psychological, social, and educational dynamics of adolescents' online social networking. Media Education. Studi, Ricerche, Buone Pratiche, 5(2), 115-130. 
Hargittai, E., \& Walejko, G. (2010). The participation divide: Content creation and sharing in the digital age. Information, Communication and Society, 11(2), 239-256.

Ivala, E., \& Gachago, D. (2012). Social media for enhancing student engagement: The use of Facebook and blogs at a university of technology. South African Journal of Higher Education, 26(1), 152-167.

Jaffar, A. A. (2012). YouTube: An emerging tool in anatomy education. Anatomical Sciences Education, 5(3), 158-164.

Jaschik, S., \& Lederman, D. (2013). The 2013 inside higher ed survey of faculty attitudes on technology. Inside Higher Ed and Gallup.

Knight, C., \& Pryke, S. (2012). Wikipedia and the university: A case study. Teaching in Higher Education, 17(6), 649-659.

Manca, S. (2014). I Social Media nell'università italiana. Diffusione degli usi personali, didattici e professionali negli Atenei italiani. Rapporto Tecnico ITD-CNR, Giugno. http://bit.ly/1 ru178f.

Manca, S., \& Ranieri, M. (2013). Is it a tool suitable for learning? A critical review of the literature on Facebook as a technology-enhanced learning environment. Journal of Computer Assisted Learning, 29(6), 487-504.

Mason, R., \& Rennie, F. (2008). E-learning and social networking handbook. New York - London: Routledge.

Moran, M., Seaman, J., \& Tinti-Kane, H. (2011). Teaching, learning, and sharing: How today's higher education faculty use Social Media. Pearson Learning Solutions and Babson Survey Research Group.

Moran, M., Seaman, J., \& Tinti-Kane, H. (2012). Blogs, wikis, podcasts and Facebook: How today's higher education faculty use Social Media. Pearson Learning Solutions and Babson Survey Research Group.

Ng'ambi, D., \& Lombe, A. (2012). Using podcasting to facilitate student learning: A constructivist perspective. Educational Technology \& Society, 15(4), 181-192.

O’Bannon, B. W., Lubke, J. K., Beard, J. L., \& Britt, V. G. (2011). Using podcasts to replace lecture: Effects on student achievement. Computers \& Education, 57(3), 1885-1892.

O'Reilly, T. (2005). What is web 2.0. Design patterns and business models for the next generation of software. http://oreilly.com/web2/archive/what-is-web-20.html.

Paulus, T., Payne, R., \& Jahns, L. (2009). «Am I making sense here?»: What blogging reveals about undergraduate student understanding. Journal of Interactive Online Learning, 8(1).

Peters, M. A. (2010). Openness, web 2.0 technology, and open science. Policy Futures in Education, 8(5), 567-574.

Ranieri, M. (2011). Le insidie dell'ovvio. Tecnologie educative e critica della retorica tecnocentrica. Pisa: ETS.

Ranieri, M., \& Manca, S. (2013a). I social network nell'educazione. Basi teoriche, modelli applicativi e linee guida. Trento: Erickson. 
Ranieri, M., \& Manca, S. (2013b). Reti professionali di insegnanti su Facebook: studio di un caso.Form@Re - Open Journal per la Formazione in Rete, 13(1), 44-54.

Ravenscroft, A. (2009). Social software, web 2.0 and learning: Status and implications of an evolving paradigm. Journal of Computer Assisted Learning, 25(1), 1-5.

Sansone, N., Cucchiara, S., \& Ligorio, M. B. (2013). L'orientamento attraverso i social network: il progetto "AAA Futuro Cercasi».Form@Re - Open Journal per la Formazione in Rete, 13(1), 81-90.

Seaman, J., \& Tinti-Kane, H. (2013). Social media for teaching and learning. Pearson Learning Solutions and Babson Survey Research Group.

Selwyn, N. (2009). Faceworking: Exploring students' education-related use of Facebook. Learning, Media and Technology, 34(2), 157-174.

Selwyn, N. (2011). Social media in higher education. In The Europan World of Learning 2012. London: Routledge.

Siemens, G. (2005). Connectivism: A learning theory for the digital age. International Journal of Instructional Technology and Distance Learning, 2(1).

Siemens, G., \& Weller, M. (2011). Higher education and the promises and perils of social networks. Revista de Universidad y Sociedad del Conocimiento, 8(1), 164-170.

Solomon, G., \& Schrum, L. (2010). Web 2.0. How-to for educators. Eugene, OR Washington, DC: International Society for Technology in Education.

Stohlmann, M. (2012). YouTube incorporated with mathematical modelling activities: Benefits, concerns, and future research opportunities. International Journal for Technology in Mathematics Education, 19(3), 117-124.

Tapscott, D., \& Williams, A. (2007). Wikinomics. New York: Atlantic.

Tinti-Kane, H., Seaman, J., \& Levy, J. (2010). Social Media in higher education. Pearson Learning Solutions and Babson Survey Research Group.

Teo, T. (2013). Online and paper-based survey data: Are they equivalent? British Journal of Educational Technology, 44(6), E196-E198.

Trentin, G. (2009). Using a wiki to evaluate individual contribution to a collaborative learning project. Journal of Computer Assisted Learning, 25(1), 43-55.

Ulrich, J., \& Karvonen, M. (2011). Faculty instructional attitudes, interest, and intention: Predictors of web 2.0 use in online courses. The Internet and Higher Education, 14(4), 207-216.

Van Zanten, R., Somogyi, S., \& Curro, G. (2012). Purpose and preference in educational podcasting. British Journal of Educational Technology, 43(1), 130-138.

Veletsianos, G., \& Kimmons, R. (2013). Scholars and faculty members' lived experiences in online social networks. The Internet and Higher Education, 16(1), 43-50. 
Weller, M. (2011). The Digital Scholar. How technology is transforming scholarly practice. London - New Dely - New York - Sydney: Bloomsbury.

Woo, M. M., Chu, S. K. W., \& Li, X. (2013). Peer-feedback and revision process in a wiki mediated collaborative writing. Educational Technology Research and Development, 61(2), 279-309.

Yu, A., Tian, S., Vogel, D., \& Kwok, R. (2010). Embedded social learning in online social networking. In ICIS 2010 Proceedings. Paper, 100. http://aisel.aisnet. org/icis2010_submissions/100.

\section{RiAsSUNTO}

L'articolo riporta i risultati di unindagine rivolta agli accademici italiani con l'obiettivo di rilevare gli usi didattici dei Social Media nel panorama delle pratiche didattiche universitarie. A questo scopo è stato tradotto e adattato un questionario, già somministrato in ambito statunitense, che è stato inviato a tutto il personale di ricerca di tutti gli atenei italiani. Hanno risposto 6.139 ricercatori/docenti universitari, pari al 10,5\% del totale. I risultati mostrano un uso ancora piuttosto limitato di questi strumenti a fronte di motivazioni di ordine e natura diversa, che evidenziano il prevalere di forme tradizionali di insegnamento, soprattutto faccia-a faccia, su pratiche più sociali e collaborative. Inoltre, emergono anche fattori di resistenza culturale o di percezione di inutilità di questi strumenti, che potrebbero contribuire a mettere in crisi i modelli tradizionali della relazione pedagogica. Una combinazione di fattori di natura educativa, pragmatica e valoriale sembrano indurre, quindi, $i$ docenti a preferire la didattica tradizionale in presenza o al più le tradizionali piattaforme e-learning. Nel complesso i risultati non possono, però, essere letti in modo univoco in quanto riflettono una ambivalenza presente anche in letteratura rispetto alle potenzialità o meno dei Social Media per l'alta formazione.

Parole chiave: Alta formazione, Pratiche didattiche, Relazione pedagogica, Social Media, Università italiana. 